University of Nebraska - Lincoln

DigitalCommons@University of Nebraska - Lincoln

\title{
Effect of Disorder on Perpendicular Magnetotransport in Co/Cu multilayers
}

Evgeny Y. Tsymbal

University of Nebraska at Lincoln, tsymbal@unl.edu

Follow this and additional works at: https://digitalcommons.unl.edu/physicstsymbal

Part of the Condensed Matter Physics Commons

Tsymbal, Evgeny Y., "Effect of Disorder on Perpendicular Magnetotransport in Co/Cu multilayers" (2000). Evgeny Tsymbal Publications. 13.

https://digitalcommons.unl.edu/physicstsymbal/13

This Article is brought to you for free and open access by the Research Papers in Physics and Astronomy at DigitalCommons@University of Nebraska - Lincoln. It has been accepted for inclusion in Evgeny Tsymbal Publications by an authorized administrator of DigitalCommons@University of Nebraska - Lincoln. 


\title{
Effect of disorder on perpendicular magnetotransport in $\mathrm{Co} / \mathrm{Cu}$ multilayers
}

\author{
E. Y. Tsymbal \\ Department of Materials, University of Oxford, Parks Road, Oxford OX1 3PH, United Kingdom
}

(Received 28 April 2000)

\begin{abstract}
We investigate the spin-dependent conductance of the $\mathrm{Co} / \mathrm{Cu}$ multilayers in the current-perpendicular-tothe-plane (CPP) geometry. Using a realistic tight-binding model for the electronic band structure of the multilayer, and introducing disorder in the on-site atomic energies we calculate the conductance and giant magnetoresistance (GMR) within the quantum-mechanical linear response theory by performing averaging over random disorder configurations. By varying the thickness and the number of individual layers and the degree of disorder in the multilayers, we analyze factors influencing the CPP GMR. In particular, we show the importance of the thickness-dependent interface resistance, which depends on the mean free path and consequently lies beyond the two-current series-resistor model.
\end{abstract}

Giant magnetoresistance (GMR) (Refs. 1 and 2) is a change in the electrical resistance that occurs in magnetic layered films when an applied magnetic field changes the relative alignment of the magnetizations of the ferromagnetic layers. This phenomenon can be observed in two principal geometries: current in the plane of the layers (CIP), ${ }^{1,2}$ and current perpendicular to the planes $(\mathrm{CPP}) .^{3}$ Although due to the small multilayer film thickness, experiments within the CPP geometry are much more delicate, they can provide important information about the mechanisms of spin-dependent scattering. Despite the large number of publications and the successful exploitation of GMR, recent experimental and theoretical results $^{4-7}$ indicate that the CPP GMR has not yet been fully understood.

Since the successful experiments on the CPP GMR have been performed, the theoretical treatment became a subject of much attention. The first theories of CPP GMR were based on free-electron models ${ }^{8,9}$ or single-band tight-binding models ${ }^{10}$ extended to magnetic layered systems. Although recent papers ${ }^{6,11}$ show that the capability of these models has not been fully exploited, calculations performed within the ballistic regime of conduction highlighted the crucial role of the electronic band structure for the CPP GMR. ${ }^{12,13}$ Including a realistic band structure within a diffusive model is related to the necessity of evaluating the vertex corrections in the configuration average of the product of two Green's functions, which is not an easy problem, especially within the first-principle methods. This is why the contribution from the vertex corrections is normally neglected, which seems to be a better approximation for the CIP transport. ${ }^{14}$ An alternative approach is making use of the real space geometry and numerically performing the averaging over random disorder configurations. ${ }^{15}$ This kind of approach was used in a recent paper $^{16}$ within a simple-cubic two-band model. Unfortunately, the conclusions about the influence of disorder on the CPP GMR, which were made in this paper, are based on considering the localization regime of conduction which is not relevant to the existing thin-film layered structures. Implementing this approach within the first-principle methods could be an important step forward in the theoretical treatment of the perpendicular magnetotransport. ${ }^{17}$
In the present paper we study the CPP GMR in the $\mathrm{Co} / \mathrm{Cu}$ multilayers using a realistic tight-binding model for the electronic structure and including defect scattering. We use a real space approach, ${ }^{15}$ which can be generalized to threedimensional multiband structures (e.g., Ref. 13). By taking a relatively large unit cell in the direction perpendicular to the current, we introduce disorder in the system which reflects the presence of intrinsic defects within the multilayer. The conductance is then calculated for each disorder configuration and the result is averaged over the random configurations. This approach differs from that of our paper, ${ }^{14}$ in which the configuration averaging is performed analytically within the weak scattering approximation that requires the inclusion of the vertex corrections for the accurate description of the CPP transport. The approach which we use in this paper goes beyond the limitations of the single-band models $^{9-11}$ and the ballistic regime of conduction. ${ }^{12,13}$

Calculations of the conductance are, therefore, performed using the Kubo formula within the real space technique, ${ }^{15}$ which is convenient for layered systems. In accordance with this approach we consider a disordered $\mathrm{Co} / \mathrm{Cu}$ multilayer stacking in the [001] direction and consisting of various number of layers of various thickness. The $\mathrm{Co}$ and $\mathrm{Cu}$ layers are assumed to have the fcc structure with a lattice parameter equal to that of bulk $\mathrm{Cu}$, i.e., $a=0.361 \mathrm{~nm}$. The multilayer is attached to the two perfect semi-infinite $\mathrm{Cu}$ leads. The electronic structure of the multilayer and the leads is treated using a realistic multiband tight-binding model accounting for $s$, $p$, and $d$ orbitals with their full hybridization and spin polarization. ${ }^{14}$ First, we find the matrix elements of the surface Green's function for the semi-infinite leads, which can be expressed in terms of the Green's function for the bulk metal (e.g., Ref. 18). Then, the $\mathrm{Co} / \mathrm{Cu}$ multilayer is grown by adding disordered layers onto the left lead. The disorder is introduced as a random variation of the on-site atomic energies of the $\mathrm{Co}$ and $\mathrm{Cu}$ atoms with a uniform distribution of standard deviation $\gamma$ which was varied in the calculations. The Green's function of the added layers is recalculated at each step recursively by solving numerically the respective Dyson equation. Once the sample has been fully grown, the last layer is bonded to the right lead in order to obtain the Green's function $\hat{G}\left(E_{F}\right)$ of the full system, which enters the 
expression for the conductance:

$$
\Gamma=\frac{\hbar}{\pi a^{2}}\left\langle\operatorname{Tr}\left[\hat{J} \operatorname{Im} \hat{G}\left(E_{F}\right) \hat{J} \operatorname{Im} \hat{G}\left(E_{F}\right)\right]\right\rangle .
$$

Here $\langle\ldots\rangle$ denotes averaging over disorder configurations, $E_{F}$ is the Fermi energy, $a$ is the lattice parameter, and the local current operator $\hat{J}$ takes the form

$$
\begin{aligned}
\hat{J}= & \frac{i e}{\hbar} \sum_{i j}\left(\mathbf{r}_{j l+1}-\mathbf{r}_{i l}\right) \mathbf{n} \sum_{\alpha \beta}\left\{h_{\beta j l+1, \alpha i l}|\beta, j, l+1\rangle\langle\alpha, i, l|\right. \\
& \left.-h_{\alpha i l, \beta j l+1}|\alpha, i, l\rangle\langle\beta, j, l+1|\right\},
\end{aligned}
$$

where $\mathbf{n}$ is a unit vector in the direction of the electric current, $|\alpha, i, l\rangle$ is the $\alpha$ orbital of the atom which lies within the layer $l$ at the in-plane site $i$ and which has coordinate $\mathbf{r}_{i l}$, and $h_{\alpha i l, \beta j l+1}$ are the tight-binding hopping integrals between planes $l$ and $l+1$. We note that $l$ can be chosen arbitrarily because of the current conservation condition. In order to introduce disorder we built a unit cell of $4 a \times 4 a$ in the transverse direction and impose periodic boundary conditions. Within the second-nearest-neighbor tight-binding model, this implies that the unit cell contains $8 \times 8$ atoms, resulting in the necessity to invert a matrix of $8 \times 8 \times 9=576$ rank at each recursion step. The conductance is calculated using a grid of $4 k$ points in the full Brillouin zone and is averaged over from 6 to 16 random configurations of disorder. This choice of parameters provides a reasonable compromise between the accuracy of calculations and the computation time.

We would like to emphasize that the size of the unit cell is a very important parameter which must be taken care of. It determines the scale of the transverse periodicity in the disordered structure and leads to the correlations in the scattering potential. Due to this, for any fixed size of the unit cell, the resistance of a disordered layer departs from the Ohmic regime and grows exponentially when the layer thickness exceeds a certain critical value $L_{c}$. Although this regime might seem to be an analogue of the localization in the disordered wires, ${ }^{19}$ in the present case it is a consequence of the artificially introduced periodicity of the scattering potential in the transverse direction. The critical thickness is determined by the number of conducting channels within the unit cell $N$ (which is proportional to the area of the unit cell) and the mean free path $l_{m f p}$, i.e., $L_{c} \sim N l_{m f p}$. The number of conducting channels in real experiments on the CPR GMR is, however, determined by the size of the multilayer in the transverse direction, i.e., it is many orders in magnitude larger than a typical value of $N$. Therefore, the effect of the localization on the CPP GMR (Ref. 20) could be relevant only to magnetic nanowires with a very small cross section. However, no experiments have yet been performed on these structures. On the other hand, making conclusions about the influence of disorder and/or the film thickness on magnetotransport in the existing thin-film layered structures by considering the "localization regime" 16 is meaningless because this regime is an artifact of the transverse periodicity constraint.

In order to be confident that we are not exceeding the critical thickness for the chosen unit cell of $4 a \times 4 a$, first, we calculated the conductance of the disordered $\mathrm{Cu}$ and $\mathrm{Co}$ metal layers (in the case of the Co layer, the Co leads were

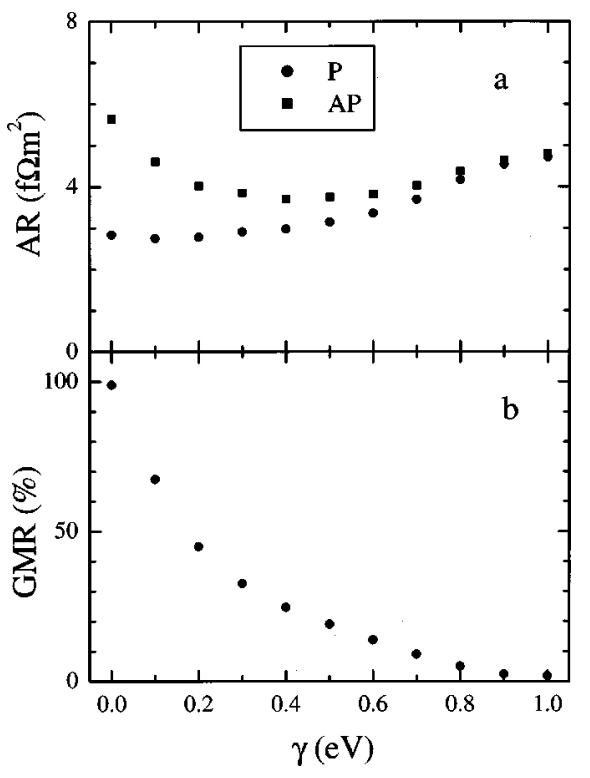

FIG. 1. Resistance of the $\mathrm{Co}_{10} / \mathrm{Cu}_{10} / \mathrm{Co}_{10}$ trilayer for parallel (P) and antiparallel (AP) magnetizations (a) and GMR (b) as a function of disorder parameter $\gamma$.

attached) as a function of their thickness and disorder $\gamma$. We found that the resistance of the $\mathrm{Cu}$ layer and both the majority- and minority-spin electrons of the Co layer display a linear behavior of up to 200 monolayers (ML) when the disorder parameter is set $\gamma=0.6 \mathrm{eV}$. Reducing the unit cell down to $2 a \times 2 a$ and $1 a \times 1 a$ resulted in an appreciable departure from the linearity, which was especially pronounced for the minority-spin electrons of Co. We found that within the Ohmic regime of conduction the increasing disorder results in a decrease in the asymmetry of the resistivity between the majority- and minority-spin electrons in $\mathrm{Co}$, which is the consequence of the interband transitions driven by the applied electric field as was explained in Ref. 14.

In the calculation of the CPP GMR for the $\mathrm{Co} / \mathrm{Cu} / \mathrm{Co}$ trilayer, the ferromagnetic Co layers were assumed to have either parallel (P) or antiparallel (AP) alignment of their magnetizations. The thickness of the layers was taken to be $10 \mathrm{ML}$ each, i.e., $3.61 \mathrm{~nm}$. Figure 1 shows the resistance and the GMR of the trilayer as a function of disorder parameter $\gamma$. As is evident from Fig. 1(b), the CPP GMR decreases with increasing disorder, which is consistent with the result of Ref. 14, but is expectedly opposite to the results predicted in Ref. 16.

An interesting feature of Fig. 1(a) is the reduction of the resistance of the trilayer with increasing disorder from 0 to $0.5 \mathrm{eV}$ for the AP configuration (squares in Fig. 1). We found that this is also the case for the minority spins within the $\mathrm{P}$ configuration (not shown). This behavior originates from the strong mismatch in the band structures of the minority-spin electrons in $\mathrm{Co}$ and $\mathrm{Cu}$, which in the absence of disorder leads to a low transmission coefficient through the $\mathrm{Co} / \mathrm{Cu}$ interface for the minority spins. ${ }^{21}$ As was shown in Ref. 22, the low transmission coefficient is the condition at which the diffuse scattering assists conduction, decreasing the resistance.

Figure 2 shows the calculated resistance and the CPP GMR for the $\mathrm{Co}_{10} / \mathrm{Cu}(t) / \mathrm{Co}_{10}$ trilayer vs $\mathrm{Cu}$ layer thickness $t$. In this calculation the disorder parameter was set equal to 


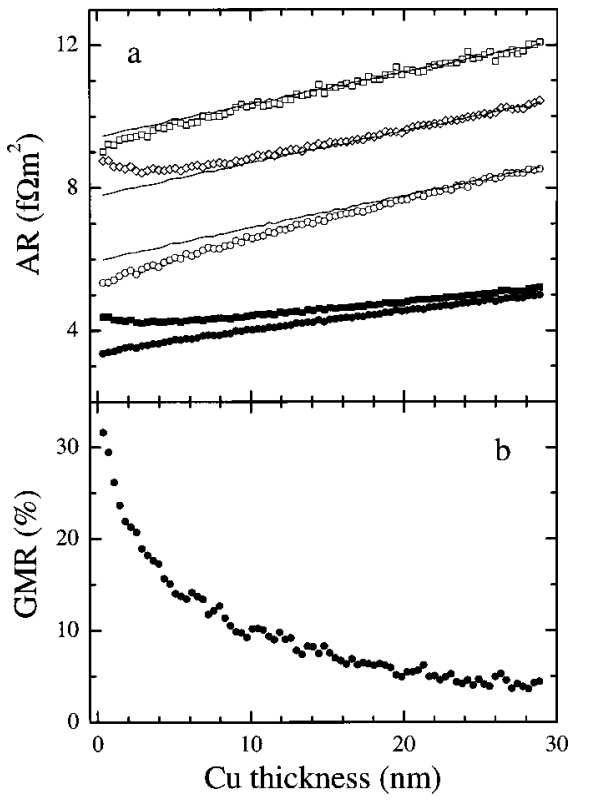

FIG. 2. Resistance (a) and GMR (b) of the $\mathrm{Co}_{10} / \mathrm{Cu}(t) / \mathrm{Co}_{10}$ trilayer as a function of $\mathrm{Cu}$ layer thickness $t$. Open symbols show the resistance per spin of the majority (circles) and minority (squares) spins for $\mathrm{P}$ magnetizations and for AP magnetizations (diamonds). Full symbols show the total resistance for $\mathrm{P}$ (circles) and AP (squares) magnetizations. Solid lines display the calculated resistance of the $\mathrm{Cu}$ layer which was scaled to fit the respective resistances for the trilayer.

$\gamma=0.6 \mathrm{eV}$, which gives the bulk resistivity of $4.6 \mu \Omega \mathrm{cm}$ for $\mathrm{Cu}$ and $14.3 \mu \Omega \mathrm{cm}$ for Co. As is seen from Fig. 2(b), the GMR decreases monotonously with $\mathrm{Cu}$ spacer thickness. Although this tendency is consistent with what one could expect from the two-current series-resistor (2CSR) model for the CPP GMR, the detailed behavior is quite different. As is evident from Fig. 2(a), the resistance of the majority-spin electrons within the $\mathrm{P}$ configuration and the resistance for the AP configuration show pronounced nonlinearity as a function of the $\mathrm{Cu}$ thickness. For comparison we plotted the calculated resistance of the disordered $\mathrm{Cu}$ layer attached directly to the $\mathrm{Cu}$ leads, which was scaled to include the resistance of the Co layers and the $\mathrm{Co} / \mathrm{Cu}$ interfaces in order to fit to the respective resistances of the $\mathrm{Co}_{10} / \mathrm{Cu}(t) / \mathrm{Co}_{10}$ trilayer at large $\mathrm{Cu}$ thickness (solid curves). A sizable deviation of the symbols from the solid lines is seen in Fig. 2(a) at small $\mathrm{Cu}$ thickness.

A key to the understanding of this behavior is the thickness-dependent interface resistance. As was demonstrated in Ref. 6, if the metal layer is placed in a potential well, quantum-well bound states reduce the number of conducting channels in this layer. This leads to the reduced interface resistance and the enhanced effective resistivity of the metal. With increasing the thickness of the disordered layer, defect scattering redistributes current-carrying electrons between various conducting channels which enhances the interface resistance. The interface resistance that is dependent on the layer thickness is the feature of the perpendicular transport which lies beyond the 2CSR model. The quantum-well states are, therefore, responsible for the nonlinear resistance of both the majority and minority electrons within the P configuration of the magnetizations [the circles and the squares

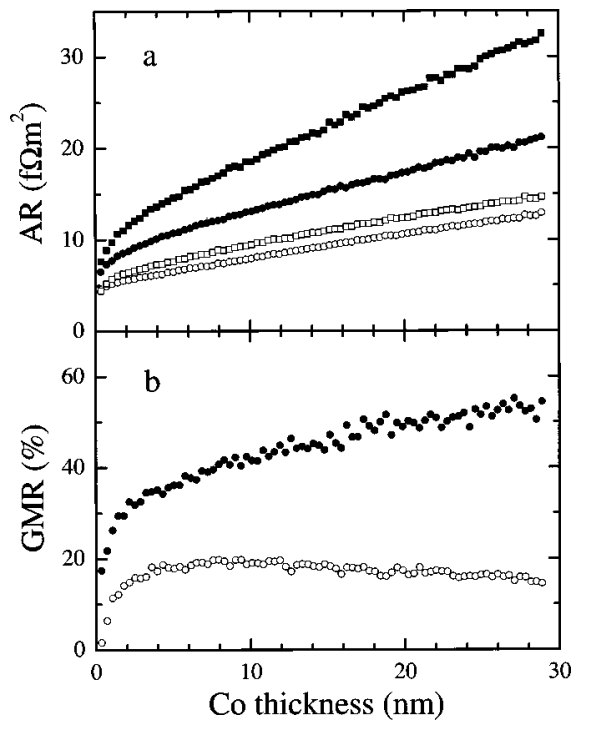

FIG. 3. Resistance for parallel (circles) and antiparallel (squares) magnetizations (a) and GMR (b) as a function of Co layer(s) thickness $t$. Open symbols: $\mathrm{Co}_{10} / \mathrm{Cu}_{10} / \mathrm{Co}(t)$ trilayer. Full symbols: $\mathrm{Co}(t) / \mathrm{Cu}_{10} / \mathrm{Co}(t)$ trilayer.

in Fig. 2(a)]. It is interesting that the minority electrons, for which one could expect a deeper potential well, display a less-pronounced departure from linearity. This is due to the effect of disorder which intermixes the closely lying Co $d$ bands and smears out the potential well. Indeed, a separate calculation in which no disorder was assumed within the Co layers demonstrated much stronger nonlinearity for the minority $\mathrm{Co}_{10} / \mathrm{Cu}(t) / \mathrm{Co}_{10}$ resistance (not shown).

The situation is, however, different for the AP configuration. As is evident from the diamonds in Fig. 2(a), in the AP case, the interface resistance is enhanced and the resistivity becomes negative at small $\mathrm{Cu}$ thickness. The effect arises from the very distinct electronic structures of the majority and minority bands in Co. In this case, scattering by disorder in the $\mathrm{Cu}$ layer assists the electrons which have passed the first $\mathrm{Co} / \mathrm{Cu}$ interface to be transmitted across the second $\mathrm{Cu} / \mathrm{Co}$ interface, reducing the interface resistance with increasing the $\mathrm{Cu}$ layer thickness.

The fact that the interface resistance is dependent on the proximity of the other interfaces is consistent with the prediction of Ref. 7, according to which the interface resistance is affected by the exponential terms in the electrochemical potential that decay at a rate comparable to the mean free path. We note that since the thickness-dependent interface resistance depends on the mean free path, measuring the nonlinear resistance as a function of the layer thickness provides a way to determine the mean free path.

Figure 3 shows the resistance and GMR as a function of the Co layer thickness. Two sets of calculations have been performed. In the first set the thickness of the first Co layer was fixed at $10 \mathrm{ML}$ and the thickness of the second Co layer was varied. In the second set the thickness of both Co layers was assumed equal and was varied. As is seen from Fig. 3(a), with increasing the Co layer thickness the resistance of the $\mathrm{Co}_{10} / \mathrm{Cu}_{10} / \mathrm{Co}(t)$ and $\operatorname{Co}(t) / \mathrm{Cu}_{10} / \mathrm{Co}(t)$ trilayers increases gradually for both the $\mathrm{P}$ and $\mathrm{AP}$ magnetizations. In the case of the $\mathrm{Co}_{10} / \mathrm{Cu}_{10} \mathrm{Co}(t)$ trilayer, at large Co thickness the total resistance of the trilayer is dominated by this Co layer, 
and therefore the difference in the resistances between the $\mathrm{P}$ and AP configurations becomes constant [compare the open circles and the squares in Fig. 3(a)]. This leads to a slow decrease of the GMR, following the saturation at about $8 \mathrm{~nm}$ [the open circles in Fig. 3(b)]. In the case of the $\mathrm{Co}(t) / \mathrm{Cu}_{10} / \mathrm{Co}(t)$ trilayer, the difference in the resistance between the $\mathrm{P}$ and AP configurations increases [compare the full circles and the full squares in Fig. 3(a)], resulting in a saturation of GMR at large Co thickness [the full circles in Fig. 3(b)]. We note that within the statistical errors the resistance and GMR do not display quantum oscillations, which are the main feature of the ballistic regime of conduction. ${ }^{13}$ These oscillations are obviously smeared out by the disorder within the trilayer.

Finally, we calculated the CPP GMR as a function of the number of the $\mathrm{Co} / \mathrm{Cu}$ bilayers $n$ in the $\left(\mathrm{Cu}_{10} / \mathrm{Co}_{10}\right)_{n}$ multilayer. As is seen from Fig. 4(a), the P and AP resistances increase in a nearly linear fashion as a function of $n$, which is evident from the fit displayed in Fig. 4(a) by the solid lines. This is exactly what one could expect from the 2CSR model. However, the reality is more complicated. The extrapolation of the linear fit to zero $n$, from which one could expect to obtain the sum of the resistances of the leads and the $\mathrm{Co} / \mathrm{Cu}$ interface, results in different resistance values for the $\mathrm{P}$ and AP magnetizations. For the $\mathrm{P}$ configuration we obtain $3.5 f \Omega \mathrm{m}^{2}$, which is by a factor of 2 higher than the value for the AP configuration $1.8 f \Omega \mathrm{m}^{2}$. This enhanced value is a consequence of the thickness-dependent interface resistance, which was discussed above. For the majority-spin electrons, which predominantly contribute to the resistance within the $\mathrm{P}$ configuration, the interface resistance is reduced for the small number of the $\mathrm{Co} / \mathrm{Cu}$ bilayers. Although the thickness-dependent interface resistance saturates very quickly, almost within two to three repeats, it results in the offset of the resistance curve origin between the P and AP configurations, as can be seen from Fig. 4(a). This leads to a very slow saturation in the CPP GMR, i.e., $\propto 1 / n$.

In conclusion, we have calculated the CPP GMR in the $\mathrm{Co} / \mathrm{Cu}$ multilayers within a realistic tight-binding model and the quantum-mechanical linear response theory. We demon-

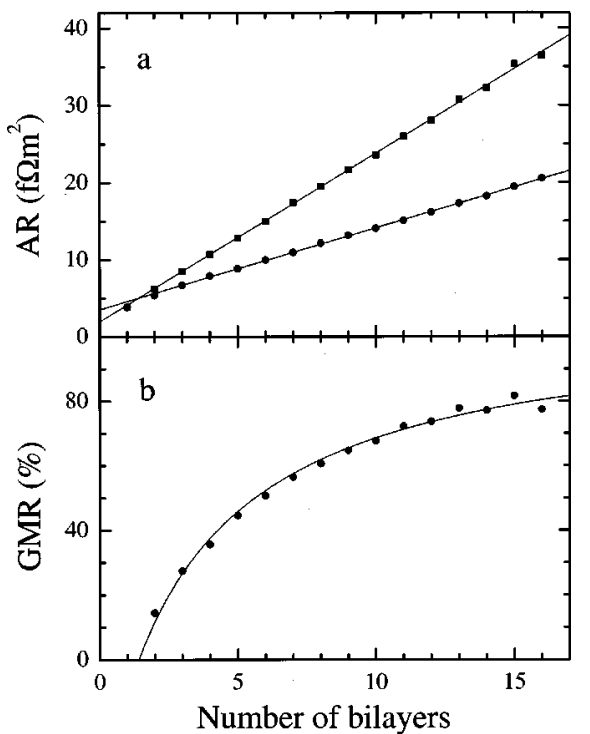

FIG. 4. Resistance of the $\left(\mathrm{Cu}_{10} / \mathrm{Co}_{10}\right)_{n}$ multilayer for the parallel (circles) and antiparallel (squares) magnetizations (a) and GMR (b) as a function of the number of bilayers $n$. Solid lines display a linear fit of the resistances and the GMR calculated from this fit.

strated the importance of the accurate account for the band structure of the multilayer which in the presence of disorder results in the thickness-dependent interface resistance. The thickness-dependent interface resistance is sensitive to the mean free path and therefore cannot be explained within the two-current series-resistor model for the CPP GMR.

This research was supported by Hewlett-Packard Laboratories in Palo Alto through a collaborative research program. The author is grateful to Didier Bozec, Bryan Hickey, Chris Marrows, and David Pettifor for helpful discussions. The computations were performed on the HP Convex Exemplar computer in the Materials Modelling Laboratory at the Department of Materials, University of Oxford, which was funded jointly by Hewlett-Packard and the Higher Education Funding Council of England.
${ }^{1}$ M. N. Baibich et al., Phys. Rev. Lett. 61, 2472 (1988).

${ }^{2}$ G. Binash et al., Phys. Rev. B 39, 4828 (1989).

${ }^{3}$ W. P. Pratt, Jr. et al., Phys. Rev. Lett. 66, 3060 (1991).

${ }^{4}$ D. Bozec et al., Phys. Rev. Lett. (to be published).

${ }^{5}$ F. Taddei et al., Phys. Rev. Lett. 82, 4938 (1999).

${ }^{6}$ E. Y. Tsymbal and D. G. Pettifor, Phys. Rev. B 61, 506 (2000).

${ }^{7}$ W. H. Butler et al., J. Supercond. 13, 221 (2000).

${ }^{8}$ S. Zhang and P. M. Levy, J. Appl. Phys. 69, 4786 (1991); H. E. Camblong et al., Phys. Rev. B 47, 4735 (1993).

${ }^{9}$ T. Valet and A. Fert, Phys. Rev. B 48, 7099 (1993).

${ }^{10}$ Y. Asano et al., Phys. Rev. B 48, 6192 (1993).

${ }^{11}$ A. Vedyayev et al., Phys. Rev. B 55, 3728 (1997).
${ }^{12}$ K. M. Schep et al., Phys. Rev. Lett. 74, 586 (1995).

${ }^{13}$ J. Mathon et al., Phys. Rev. B 55, 14378 (1997).

${ }^{14}$ E. Y. Tsymbal and D. G. Pettifor, Phys. Rev. B 54, 15314 (1996).

${ }^{15}$ P. A. Lee and D. S. Fisher, Phys. Rev. Lett. 47, 882 (1981).

${ }^{16}$ S. Sanvito et al., Phys. Rev. B 60, 7385 (1999).

${ }^{17}$ J. Kudrnovský et al., Phys. Rev. B (to be published).

${ }^{18}$ J. Pollmann and S. T. Pantelides, Phys. Rev. B 18, 5524 (1978).

${ }^{19}$ P. W. Anderson, Phys. Rev. B 23, 4828 (1981).

${ }^{20}$ J. Mathon, Phys. Rev. B 55, 960 (1997).

${ }^{21}$ M. Stiles, J. Appl. Phys. 79, 5805 (1996).

${ }^{22}$ S. Zhang and P. M. Levy, Phys. Rev. B 57, 5336 (1998). 